\title{
Precision Cardio-Oncology
}

\author{
Alexandra D. Dreyfuss ${ }^{1}$, Paco E. Bravo ${ }^{2,3}$, Constantinos Koumenis ${ }^{1,4}$, and Bonnie Ky ${ }^{3,4}$ \\ ${ }^{1}$ Department of Radiation Oncology, Perelman School of Medicine, University of Pennsylvania, Philadelphia, Pennsylvania; \\ ${ }^{2}$ Division of Nuclear Medicine, Department of Radiology, University of Pennsylvania, Philadelphia, Pennsylvania; ${ }^{3}$ Division \\ of Cardiology, Department of Medicine, University of Pennsylvania, Philadelphia, Pennsylvania; and ${ }^{4}$ Abramson Cancer Center, \\ University of Pennsylvania, Philadelphia, Pennsylvania
}

\begin{abstract}
Modern oncologic therapies and care have resulted in a growing population of cancer survivors with comorbid, chronic health conditions. As an example, many survivors have an increased risk of cardiovascular complications secondary to cardiotoxic systemic and radiation therapies. In response, the field of cardio-oncology has emerged as an integral component of oncologic patient care, committed to the early diagnosis and treatment of adverse cardiac events. However, as current clinical management of cancer therapyrelated cardiovascular disease remains limited by a lack of phenotypic data, implementation of precision medicine approaches has become a focal point for deep phenotyping strategies. In particular, -omics approaches (a field of study in biology ending in -omic, such as genomics, proteomics, or metabolomics) have shown enormous potential in identifying sensitive biomarkers of cardiovascular disease, applying sophisticated, pattern-revealing technologies to growing databases of biologic molecules. Moreover, the use of -omics to inform radiologic strategies may add a dimension to future clinical practices. In this review, we present a paradigm for a precision medicine approach to the care of cardiotoxin-exposed cancer patients. We discuss the role of current imaging techniques; demonstrate how -omics can advance our understanding of disease phenotypes; and describe how molecular imaging can be integrated to personalize surveillance and therapeutics, ultimately reducing cardiovascular morbidity and mortality in cancer patients and survivors.
\end{abstract}

Key Words: cardio-oncology; precision medicine; precision cardiooncology; -omics; cardiotoxicity

J Nucl Med 2019; 60:443-450

DOI: 10.2967/jnumed.118.220137

$\mathbf{T}$

he last decade has witnessed a revolution in cancer therapeutics, producing a population of cancer survivors now exceeding 15.5 million in the United States alone (1). Although encouraging, this gain in cancer survivorship has also produced a growing cohort of patients with increased cardiovascular complications. Cardiovascular disease (CVD) currently accounts for approximately $32 \%$ of deaths globally, with a significant percentage in those with previous cancer treatment (2). To address this growing public health burden, a new discipline-cardio-oncology—dedicated to the cardiovascular care of cancer patients has emerged. However, despite

Received Sep. 7, 2018; revision accepted Dec. 29, 2018.

For correspondence or reprints contact: Bonnie Ky, Division of Cardiology, Department of Medicine, University of Pennsylvania, 3400 Civic Center Blvd., Philadelphia, PA 19104.

E-mail: bonnie.ky@uphs.upenn.edu

Published online Jan. 17, 2019.

COPYRIGHT (C 2019 by the Society of Nuclear Medicine and Molecular Imaging. substantial progress made in elucidating mechanisms of therapyrelated cardiotoxicity, personalized diagnostic and therapeutic strategies remain limited, with few distinctions made between underlying mechanisms of CVD.

One strategy that has gained momentum in cardiology and oncology is the advancement of precision medicine, which relies on computational integration of clinical data with advanced genetic, biomolecular, and demographic profiling to better risk-stratify patients. This process, known as deep phenotyping, directly undermines the reductionist assumption that similar phenotypes arise from similar underlying pathophysiology. In the case of cancer therapy-related CVD, this reductionist assumption remains the current therapeutic standard, largely because of a lack of phenotyping data in risk stratification and disease development. As the link between cancer therapeutics and CVD continues to heterogeneously manifest in the clinic, our understanding of the potential applications of precision medicine in cardio-oncology is central to actualizing successful preventative, diagnostic, and therapeutic interventions.

In this review, we present a paradigm for how to advance our understanding of the disease phenotype in cancer patients exposed to potential cardiotoxins. We start with a background on the state of cardio-oncology and follow with a discussion of -omics (a field of study in biology ending in -omic, such as genomics, proteomics, or metabolomics) approaches to enhance disease characterization and elucidate novel biomarkers. We then illustrate how a variety of current and emerging imaging techniques are differentially suited for phenomapping disease. We conclude by using RT-induced microvascular injury as a case study for how -omics identification of molecular imaging targets could shape the future of personalized cardio-oncology.

\section{BACKGROUND}

Clinical manifestations of cancer therapy cardiotoxicity are heterogeneous. The most recent European Society of Cardiology guidelines broadly divide cardiovascular complications of cancer therapy into 9 categories, including myocardial dysfunction, coronary artery disease, valvular heart disease, arrhythmias, and pericardial diseases (3). The heterogeneity in cardiovascular complications of cancer therapies can, in part, be attributed to the increasing number of cancer therapeutics used in clinical practice, each with unique pathogeneses. These include traditional chemotherapeutics such as anthracyclines, targeted therapies with trastuzumab or antiangiogenic tyrosine kinase inhibitors, and immunotherapies. The importance of early detection of cardiotoxicity has become increasingly apparent; left ventricular ejection fraction (LVEF) recovery and cardiac event reduction have been shown to occur more frequently with early diagnosis and treatment of cardiotoxicity in patients with 
cancer therapy-induced cardiomyopathy (4). Accordingly, recommendations for managing potential cardiac dysfunction in patients receiving cardiotoxic therapies include LVEF monitoring with serial echocardiograms, population stratification by treatments received and preexisting cardiovascular risk factors (e.g., age, hypertension), and surveillance with serum cardiac biomarkers (troponin) (3). Additionally, a staging system (stages A-D) for heart failure exists, spanning from any cardiotoxin-exposed patient (stage A) to those with observed, asymptomatic left ventricular dysfunction (stage B) and symptomatic heart failure (stages C and D) (5).

Although such guidelines represent a step forward, recommendations are generally vague, and a deficiency in evidence-based data for clinical decision making remains. Interestingly, the limitations of current approaches highlight the multiple features that make the field of cardio-oncology uniquely suited for precision medicine. For example, current risk stratification schemes include only basic clinical characteristics and do not incorporate the wealth of information potentially offered by deep phenotyping. Even with the potential distinct categorizations that may emerge from current risk stratification models, differences in clinical management remain largely limited to adjustments in surveillance frequency and medication dosing. Moreover, more precise characterization of disease with refined imaging measures and sensitive biomarkers represents relatively untapped tools with which to tailor treatments to underlying pathophysiologic differences in otherwise similar clinical presentations. Going forward, a multidisciplinary commitment to precision medicine approaches is needed, with molecular imaging and -omics sciences at the forefront of promising archetypes.

\section{PANOMICS: BIOMARKER IDENTIFICATION}

The value of deep phenotyping has been demonstrated in studies of common CVDs (6). A natural extension of such studies has been to embrace principles of more precise clinical phenotyping in cardio-oncology. Consequently, recent research has focused on identifying circulating biomarkers of disease as a complementary approach to the pursuit of sensitive imaging metrics.

To date, most investigations have focused on troponin and brain natriuretic peptide, two well-established biomarkers of cardiac injury and stress whose elevated levels have been associated with cardiotoxicity $(7,8)$. However, it still remains to be determined if systematic evaluation of these biomarkers improves outcomes, and there are currently no guidelines for cutoffs, timing of assessments, or therapy-specific considerations. These limitations compounded by a lack of biomarker specificity have motivated a search for more evidence-based biomarkers. For example, myeloperoxidase, growth differentiation factor-15, and asymmetric dimethylarginine have all been postulated as potentially useful biomarkers, as all play critical roles in oxidative stress or nitric oxide

\section{NOTEWORTHY}

- The recent gains in cancer survivorship have produced a growing cohort of patients with increased cardiovascular complications and led to the field of cardio-oncology.

- Within cardio-oncology, current risk stratification schemes primarily include only basic clinical characteristics.

- Refined imaging measures and sensitive biomarkers of disease represent tools with the potential to tailor treatments to underlying pathophysiologic differences in patients with otherwise similar clinical presentations. metabolism - potential mechanisms of anthracycline-induced cardiotoxicity $(9,10)$. Additionally, a multimarker approach aimed at increasing sensitivity and specificity through the simultaneous evaluation of multiple contributory pathways has also been proposed, with elevations in troponin and myeloperoxidase demonstrating significant additive predictive value (9).

More recently, advancements in -omics technologies have amplified the clinical relevance of phenotyping in both magnitude and spectrum. -Omics sciences seek to computationally identify patterns from sizeable networks of data to elucidate causative pathophysiology. In the case of therapy-induced cardiotoxicity, these sciences can elucidate novel disease metrics that can inform the personalization of medicine in cardio-oncology (Fig. 1). However, it is important to note the inherent limitation of a systems-based analysis that is not specific to the heart, for predicting cardiac effects. Although -omic technologies applied in broad discovery studies may reveal measurable changes in select candidate markers, the lack of specificity of these markers for cardiac tissue must be considered; abnormalities in variables may be due to processes unrelated to the cardiovascular system. Thus, recognition of potential confounding variables has an important function in interpreting results of panomics for biomarker identification.

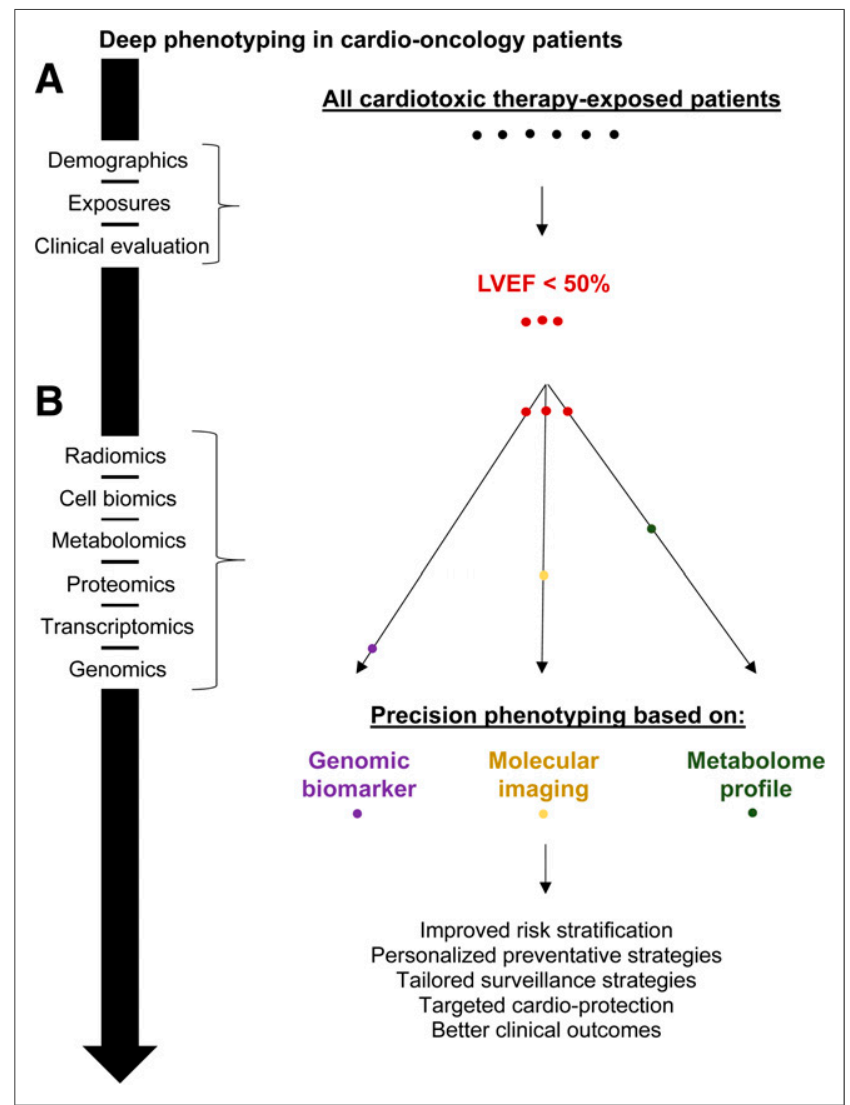

FIGURE 1. (A) Current approaches to cardio-oncology patients involve phenotyping with demographics, exposure history, and clinical evaluation (including basic lab tests and imaging). This method identifies disease phenotypes such as decreased LVEF to broadly guide therapeutic management. (B) Individuals with same phenotype (e.g., LVEF $<50 \%$ ) can be further stratified through precision phenotyping and applying -omics to elucidate defining biologic data (e.g., genomic biomarker, molecular imaging, metabolome profile), resulting in more targeted management and better outcomes. 


\section{Genomics}

Much of our understanding of the genetics of anthracyclineinduced cardiotoxicity has come from genomewide association studies of childhood survivors, which have implicated singlenucleotide polymorphisms in carbonyl reductase and hyaluronan synthase 3 as independent modifiers of anthracycline-related cardiomyopathy risk (11). CBRs catalyze the reduction of anthracyclines to cardiotoxic alcohol metabolites, whereas the hyaluronan synthase 3 gene encodes for hyaluronan, a ubiquitous component of the extracellular matrix that plays a role in tissue response to injury. Additional studies on childhood cancer survivors have identified polymorphisms in genes that regulate intracellular transport (SLC28A3, SLC28A1) of anthracyclines as independent predictors of cardiomyopathy risk $(12,13)$. A study of adult hematopoietic cell transplantation patients treated with anthracyclines identified an association between a polymorphism in the doxorubicin efflux transporter and cardiotoxicity (14). Another genomewide association study of anthracycline cardiotoxicity suggested a susceptibility locus at chromosome 1p36.21 near PRDM2 $\left(P=6.5 \times 10^{-7}\right)(15)$. Additionally, genetic polymorphisms in the HER2 gene (Ile 655 Val and Pro 1170 Ala) have also been identified as conferring increased risk for trastuzumab cardiotoxicity (16).

Circulating micro-RNA, a direct product of genomic profiles, also has significant potential for identifying subclinical cardiac damage in patients receiving certain therapies. This large class of noncoding small RNAs that circulate in the bloodstream, enter distant recipient cells, and regulate gene expression has already demonstrated potential as a biomarker of CVD. In a case-control study of 12 children, 84 micro-RNAs were profiled $24-48 \mathrm{~h}$ and approximately $1 \mathrm{y}$ after initiation of anthracycline chemotherapy and showed an association between decreased LVEF and 3 specific micro-RNAs (17). In another study of 33 children receiving anthracycline chemotherapy and noncardiotoxic chemotherapy, profiling of 24 micro-RNAs at pre- and postcycle time points revealed greater chemotherapyinduced dysregulation in patients receiving anthracyclines than in those receiving noncardiotoxic chemotherapy (24-h multivariate ANOVA, $P=0.024$ ) (18).

\section{Proteomics}

A similar and parallel approach has been implemented in biomarker identification using proteomics. In experiments on heart tissue samples from control rats and rats exposed to docetaxel and doxorubicin, 9 proteins involved in energy production were found to be differentially expressed in control versus treatment groups, with higher levels of glyceraldehyde-3-phosphate-dehydrogenase associated with lower mortality (19). The importance of energy metabolism was also confirmed in daunorubicin-induced cardiotoxicity, with alterations in mitochondrial proteins involved in oxidative phosphorylation and energy channeling along with increased proteins involved in autophagy, membrane repair, and apoptosis (20). Small human studies have also suggested the importance of the immune system in the pathophysiology of cardiotoxicity (21). Nevertheless, findings from proteomic studies are still largely preliminary.

\section{Metabolomics}

Variation in metabolite profiles uniquely reflects a spectrum of molecular influences, intricately linking changes in DNA sequences, cellular physiology, and environmental factors. Despite a lack of studies investigating metabolic changes specific to cancer therapyrelated CVD, metabolomics has emerged as a potential tool for cardio-oncologists to use in characterizing chemical intermediates in a variety of biosamples, especially as abnormal cardiac metabolism has been increasingly linked to CVD. For cardio-oncology patients, metabolic studies of general CVD offer 2 main insights: an understanding of the pathophysiologic, metabolomic alterations that occur in specific disease states (i.e., heart failure and myocardial ischemia), and a potential approach to CVD risk prediction with novel biomarkers. For example, the sequential use of progressively macroscopic-omics, that is, from genomics to clinical phenotyping, as an approach to modeling and tracking the course of a disease may be promising. This approach directly contrasts current paradigms in which changing clinical phenotypes are what motivate investigation into progressively microscopic pathophysiology, that is, from clinical chemistry and imaging to genomics.

With respect to risk prediction, the ability to one day perform realtime monitoring of blood or urine metabolites could allow clinicians to detect novel molecular biomarkers associated with differential clinical trajectories. Similarly, changes in metabolite profiles over time, such as after administration of a drug, could be used to define an individual's response to therapy and guide subsequent management.

\section{PHENOMAPPING WITH IMAGING}

The varying clinical manifestations and unpredictable timing of treatment-related CVD have made surveillance and diagnosis challenging. One attractive approach has been to use sensitive imaging measures for phenomapping the risk of developing subclinical and overt disease. Current strategies rely almost exclusively on measurements of LVEF; however, a lack of consensus criteria for meaningful cutoffs, inherent variability in LVEF values and assessment, and an inability to detect subclinical myocardial damage limit LVEF as a gold standard. The ensuing discussion focuses on prominent imaging indices used clinically and evaluates how emerging strategies may offer more comprehensive phenotypic data.

\section{LVEF}

LVEF is the most commonly used measure of cardiac function in cancer patients. Two-dimensional (2D) echocardiography is typically the modality of choice in measuring LVEF, as it is inexpensive, is easily available, and avoids radiation exposure. However, concern over its reproducibility and inability to detect small decreases in LVEF have motivated many to explore advanced techniques including strain analyses, ultrasound-based contrast agents (recommended with suboptimal 2D echocardiography views), and 3-dimensional (3D) echocardiography. A recent prospective study comparing 2D echocardiography and 3D echocardiography in breast cancer patients found that temporal variability in LVEF decreased from $10 \%-13 \%$ with 2D echocardiography to $5 \%-6 \%$ with 3D echocardiography (22). Variability in LVEF measurement with 3D echocardiography is therefore more comparable to that with cardiac MRI (CMR) (2\%) and was proposed as the preferred modality by several organizations (23). Still, because of its operator dependence, decreased availability, and increased cost, routine clinical use of 3D echocardiography has been challenging.

Multigated acquisition has also been used for LVEF assessment. In one of the largest studies using serial multigated acquisition, decreases in LVEF to less than $50 \%$, or by $10 \%$ from baseline, predicted heart failure in $19 \%$ of patients (24). Additionally, in a clinical trial involving 944 breast cancer patients treated with chemotherapy, a decreased LVEF by multigated acquisition independently predicted cardiac events over a 7-y follow-up period (25). However, the lack of information beyond LVEF and radiation exposure have limited the use of multigated acquisition. 


\section{Myocardial Deformation and Mechanics}

Myocardial deformation, as measured by echocardiographic tissue Doppler imaging or speckle-tracking echocardiography (STE), which uses a computerized algorithm to track natural echocardiographic signals, has recently emerged as a novel imaging measure. Deformation can be characterized by strain or strain rate (a measure of longitudinal, radial, and circumferential dimensions in peak systole), twist, or torsion. A major appeal of this approach comes from evidence that changes in strain can be observed before changes in LVEF and can predict cardiotoxicity (26).

Most recently, 3D STE has been proposed as a new modality to track linear myocardial deformation in multiple dimensions simultaneously, torsion, and mechanical desynchrony. Initial results have demonstrated advantages over 2D STE, including faster image acquisition, improved accuracy, and more complete analysis (27). Additionally, myocardial mechanics, including ventricular-arterial coupling and vascular stiffness, have been shown to predict declines in LVEF (28). Pending determination of optimal test timing and cutoffs, along with further validation in large cohort studies, myocardial deformation and mechanics parameters may become key components of phenotyping in cancer-associated CVD.

\section{CMR Imaging Metrics}

CMR is an effective method for identifying CVD in cancer patients. The high spatial and temporal resolution and reproducibility of CMR enable the identification of inflammatory or infiltrative processes, abnormal myocardial masses, and pericardial or valvular abnormalities, elucidating potential etiologies for reduced LVEF. One approach to characterizing cardiac tissue is to use CMR T1-weighted images and T1 mapping to detect myocardial inflammation, extent and topography of fibrosis, and pericardial tumor invasion with late gadolinium enhancement (29). Studies have demonstrated abnormal T1 signal in patients who received cardiotoxic chemotherapy, postulated to be due to either an increase in extracellular distribution volume or enhanced water exchange elicited by myocardial injury $(30,31)$. Increases in extracellular volume fraction and total extracellular volume have also been observed in small studies of patients receiving anthracycline chemotherapy, potentially suggestive of edema and fibrosis. However, the mechanistic basis for this increase in extracellular volume fraction was recently challenged in a study of patients who received anthracycline chemotherapy, with results suggesting that increases in myocardial extracellular volume fraction may not necessarily be due to expansion of interstitial space but may actually be related to decreases in myocellular volume and mass (32). T2 mapping has similarly demonstrated utility in detecting subclinical cardiotoxicity, as it enables qualitative and quantitative assessment of water content, which may increase after myocellular or microvascular injury.

CMR also has a role in assessing cardiac function and blood flow. For example, cine white-blood steady-state free precession imaging allows for evaluation of wall motion abnormalities and calculation of volumes, LVEF, and mass (29). CMR can also be used to quantify myocardial strain by either spatial modulation of magnetization or displacement encoding with stimulated echoes techniques. In a prospective study of 53 cancer patients, CMRmeasured global left ventricular circumferential strain declined in parallel with declines in LVEF after treatment with anthracyclines (33). Further studies corroborating prognostic and diagnostic value along with considerations of cost and availability are warranted and will likely be necessary before widespread implementation in clinical practice can occur.

\section{Myocardial Perfusion Imaging}

A common cardiovascular complication of cancer therapies is impairment of the coronary circulation either through direct vascular damage or accelerated atherosclerosis. As such, noninvasive methods for evaluating myocardial perfusion with such parameters as myocardial blood flow and coronary flow reserve (CFR) quantification are desirable in cardio-oncology care. For years, SPECT imaging has been one of the principal methods for evaluation of flow-limiting coronary stenosis in cardio-oncology patients, with the most commonly used radiotracers being ${ }^{99 \mathrm{~m}} \mathrm{Tc}$-labeled sestamibi and tetrofosmin. Several SPECT-based studies have observed a high incidence of new resting myocardial perfusion deficits ( $\leq 60 \%$ in some series) as early as 6 mo after radiation therapy (RT) in patients receiving left breast/chest wall RT compared with pre-RT SPECT scans $(34,35)$. Importantly, these changes in perfusion are typically limited to the anterior wall and apex or follow the typical distribution of the left anterior descending coronary artery (Fig. 2) (35), correlate with the volume of heart irradiated (36), remain relatively unchanged at 12- and 18-mo of follow-up compared with 6 mo after RT (35), and correlate with cardiovascular symptoms in those with new perfusion defects (37).

More recently, cardiac PET has witnessed more widespread clinical implementation and is the current gold standard to assess myocardial perfusion because of its higher spatiotemporal resolution, count sensitivity, and accuracy when compared with SPECT. Additionally, PET tracers including ${ }^{13} \mathrm{NH}_{3}$ and ${ }^{82} \mathrm{Rb}$ have superior pharmacokinetic properties due to a greater myocardial net uptake rate at higher coronary flows than is the case with their SPECT counterpart (38) and, combined with dynamic imaging, allow for noninvasive quantification of myocardial blood flow at baseline and during pharmacologic hyperemia. Stress myocardial blood flow is typically impaired in the presence of flow-limiting coronary stenosis; however, in its absence, impaired stress myocardial blood flow, and thereby CFR, is considered a robust marker of coronary microvascular dysfunction. Yet, limited clinical studies have applied cardiac PET to monitor for cancer therapy-related

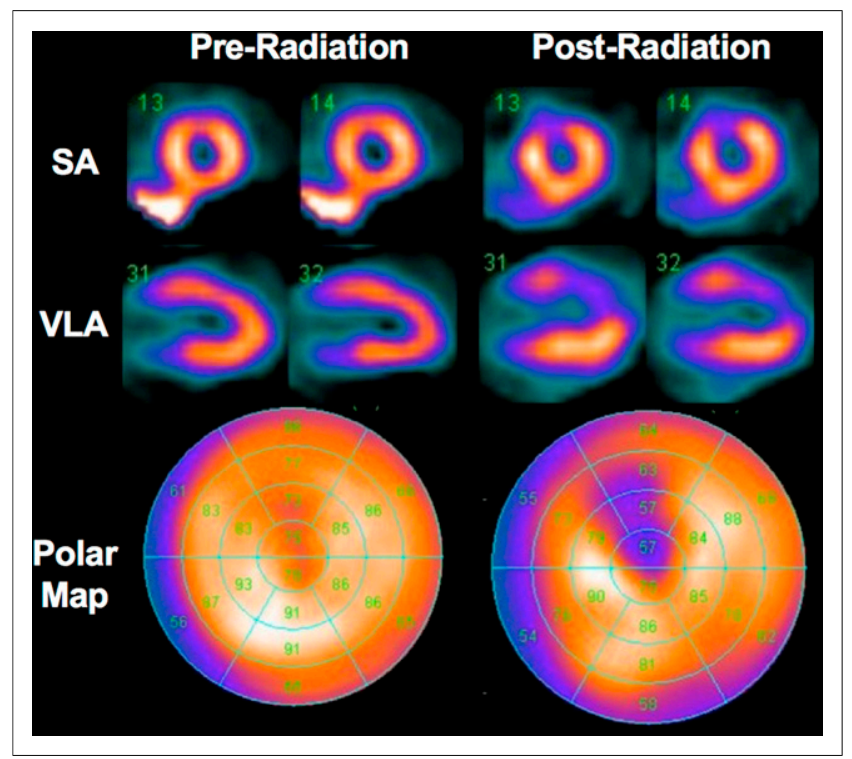

FIGURE 2. Myocardial perfusion SPECT images before and 6 mo after RT for left-sided breast cancer demonstrate development of new perfusion deficit in anterior wall and apex consistent with radiation-induced myocardial damage. SA = short axis; VLA = vertical long axis. 
cardiotoxicity. In one of the few human studies applying PET in cardio-oncology, a cross-sectional analysis of 35 patients who underwent ${ }^{82} \mathrm{Rb}$ PET/CT at a median interval of $3.6 \mathrm{y}$ after RT found significant inverse correlations between CFR and specific RT dosevolume metrics, including a significant inverse correlation between global left ventricular CFR and increasing mean heart RT dose $(R=-0.4, P=0.03)$, and regionally between CFR of and radiation dose to the left anterior descending artery $(R=-0.5, P=$ 0.002) (39).

Despite these advancements, several challenges have limited the use of these PET imaging techniques in cardio-oncology patients. For one, limited data are available with regard to optimal thresholds used to distinguish pathologic from normal hyperemic myocardial blood flow and CFR. Additionally, optimal thresholds may vary depending on radiotracer and software. Lastly, human clinical trials are needed to assess the diagnostic and prognostic value of myocardial perfusion imaging parameters before clinical implementation can occur.

\section{Cardiovascular Molecular Imaging Techniques}

Molecular imaging exploits radiolabeled imaging probes to elucidate the biomolecular events that underlie clinical phenotypes. In the field of cardiology, molecular imaging has demonstrated years of clinical utility and value, with both SPECT and PET at the center of many diagnostic approaches. Recently, such imaging techniques have demonstrated the potential to play a similar role in the field of cardio-oncology. The radiotracers, mechanisms of uptake, and targets for imaging cancer-related cardiotoxicity can be found in Table 1.

Metabolic imaging with ${ }^{18} \mathrm{~F}-\mathrm{FDG}$ PET/CT remains the backbone of molecular imaging. ${ }^{18} \mathrm{~F}-\mathrm{FDG}$ is a sensitive molecular probe for the investigation of cancer-related cardiotoxicity because tissue injury (40), inflammation (41), and hypoxia/ischemia (42) are potent stimuli for glucose transporter expression. In a canine model of radiation-induced cardiotoxicity, focal myocardial ${ }^{18} \mathrm{~F}-\mathrm{FDG}$ uptake (typically in the anterior wall) corresponding to the irradiated field was observed 3 mo after RT (Fig. 3) (40). On histopathology, all dogs displayed areas of myocardial damage in the irradiated field consisting of perivascular fibrosis and mild myocyte degeneration and mitochondria injury, but interestingly, no inflammatory cell infiltration was detected, implying that ${ }^{18} \mathrm{~F}-\mathrm{FDG}$ accumulation within the irradiated field was mediated through mechanisms other than inflammation, including tissue hypoxia/ischemia due to microvascular damage or changes in metabolism caused by mitochondrial injury (40). Similar observations have been made in a retrospective study of 39 lung cancer patients treated with RT (Fig. 4), in which $47 \%$ of patients receiving 20 Gy to at least $5 \mathrm{~cm}^{3}$ of the heart developed increased ${ }^{18} \mathrm{~F}-\mathrm{FDG}$ uptake versus $0 \%$ of the patients who received $20 \mathrm{~Gy}$ to less than $5 \mathrm{~cm}^{3}(P=0.02)$, again supporting the role of ${ }^{18} \mathrm{~F}-\mathrm{FDG}$ as a marker of RT-induced myocardial injury (43).

On the other hand, with the development of several new molecular imaging agents, many of which target early markers of cardiotoxicity (sympathetic nerve terminals, angiogenesis, reactive oxygen species, and apoptosis), the potential impact of molecular imaging on patient outcomes in cardio-oncology has become even greater. In one study of rats treated with anthracycline, PET imaging with ${ }^{18} \mathrm{~F}$ DHMT, a marker of reactive oxygen species, revealed an elevation in cardiac superoxide production at $4 \mathrm{wk}$ after treatment, compared with a decrease in LVEF on echocardiography detectable only at 6 wk after treatment (44). In one human study of 20 breast cancer patients treated with either anthracycline and trastuzumab or anthracycline alone, the ratio of ${ }^{123}$ I-metaiodobenzylguanidine uptake between the heart and mediastinum on scintigraphic images decreased in $25 \%$ of patients, and the washout rate increased in $82 \%$ of patients compared with matched controls, demonstrating the potential utility

TABLE 1

Radiotracers, Mechanisms of Uptake, and Targets for Imaging Cancer-Related Cardiotoxicity

\begin{tabular}{|c|c|c|}
\hline Radiotracer & Molecular target/mechanism of uptake & Cardiovascular application \\
\hline${ }^{82} \mathrm{Rb}^{*}$ & $\mathrm{Na} / \mathrm{K}-\mathrm{ATP}$ ase & Perfusion/flow quantification \\
\hline${ }^{13} \mathrm{NH}_{3}{ }^{*}$ & Glutamine synthetase activity & Perfusion/flow quantification \\
\hline 99mTc-sestamibi* & Mitochondrial function & Perfusion \\
\hline${ }^{18} \mathrm{~F}-\mathrm{FDG}^{\star}$ & Glucose transporters & Glucose metabolism \\
\hline${ }^{123} \mathrm{I}-\mathrm{MIBG}^{*}$ & Norepinephrine transporter & Sympathetic nerve integrity \\
\hline${ }^{11} \mathrm{C}$-hydroxyephedrine & Norepinephrine transporter & Sympathetic nerve integrity \\
\hline${ }^{11} \mathrm{C}$-acetate & Krebs cycle flux & Oxidative metabolism \\
\hline 123I-BMIPP & a-oxidation and $\beta$-oxidation & Fatty acid metabolism \\
\hline${ }^{111}$ In-trastuzumab & HER2 & Apoptosis \\
\hline${ }^{111}$ In-antimyosin & Exposed myosin & Cellular necrosis \\
\hline 99mTc-RP805† & Activated MMPs & Remodeling \\
\hline${ }^{18} \mathrm{~F}-\mathrm{DHMT}{ }^{\dagger}$ & Reactive oxygen species & Cytotoxicity \\
\hline 99mTc-annexin $\mathrm{V}^{\dagger}$ & Exposed phosphatidylserine & Apoptosis \\
\hline${ }^{18} \mathrm{~F}-\mathrm{CP} 18^{\dagger}$ & Caspase 3 activity & Apoptosis \\
\hline \multicolumn{3}{|c|}{$\begin{array}{l}{ }^{\star} \text { FDA-approved for clinical use. } \\
{ }^{\dagger} \text { Preclinical phase. No published human data on cardiotoxicity. } \\
\text { MIBG = metaiodobenzylguanidine; BMIPP }=\beta \text {-methyl-p-iodophenylpentadecanoic acid; HER } 2 \text { = human epidermal growth factor } \\
\text { ceptor 2; MMP = matrix metalloproteinases }\end{array}$} \\
\hline
\end{tabular}




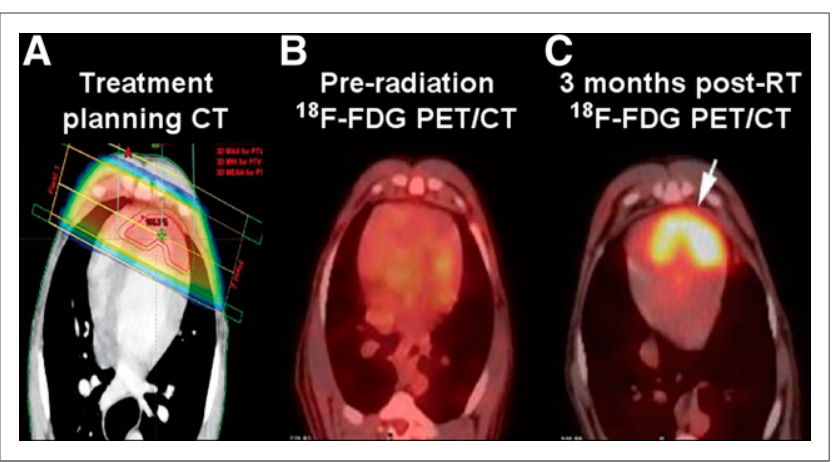

FIGURE 3. Radiation-induced myocardial injury in dogs. (A) Dose distribution contrast-enhanced cardiac CT. (B) ${ }^{18} \mathrm{~F}-\mathrm{FDG}$ PET/CT showing no myocardial uptake before RT. (C) Focally intense ${ }^{18} \mathrm{~F}-\mathrm{FDG}$ uptake along left ventricular apex matching irradiated field 3 mo after RT. (Adapted with permission of (40).)

of ${ }^{123}$ I-metaiodobenzylguanidine as a marker of cardiac sympathetic activity and cardiotoxicity (45). However, in a more recent study of 89 asymptomatic patients previously treated with anthracyclines, neither the heart-to-mediastinum ratio nor the washout rate was able to discriminate anthracycline-exposed patients from controls (46).

Because molecular imaging techniques with newer probes are still under study, the role for these approaches remains to be defined. Still, molecular imaging innovations occurring concurrently with those in -omics technologies have the potential to reveal radiologically targetable, novel molecular biomarkers. Thus, a next step in cardio-oncology care could involve the coupling of biomarker discovery with radiographic technologies to capture real-time molecular events, ultimately creating a key role for nuclear medicine in the future of cardio-oncology.

\section{RADIATION-INDUCED CARDIOTOXICITY}

Radiation-induced CVD represents a nuanced clinical challenge. A recent retrospective study reported that compared with nonirradiated patients, chest RT patients have a $2 \%$ and $23 \%$ higher absolute risk of cardiac morbidity and death at 5 and $20 \mathrm{y}$ after treatment, respectively (47). In contrast to chemotherapy-induced toxicity, which is largely dependent on metabolic rates or genetic differences in drug metabolism, RT toxicity can be directly linked to received dose to the heart or nearby organs. However, in line with novel precision medicine strategies for drug-induced CVD, radiation oncologists have proposed therapeutic approaches such as the use of gene-expression assays to identify potential singlenucleotide polymorphisms that may predict adverse reactions to RT and guide radiation dosing guidelines-a concept known as the genomic-adjusted radiation dose (48). Furthermore, because cardiovascular radiation damage is postulated to be caused in part by monocyte activation and the formation of inflammatory plaque and intimal fatty streaks - major components of atherosclerosisleading to vessel occlusion, perfusion defects, and myocardial necrosis and fibrosis, RT patients are uniquely suited for surveillance and risk stratification with radionuclide imaging techniques.

\section{Radiation-Induced Atherosclerosis}

A potential approach to early diagnosis and surveillance of radiation-induced atherosclerosis is to use molecular imaging to predict likely outcomes based on molecular evaluation of plaque rupture propensity. For example, PET imaging of plaque macrophages with ${ }^{18} \mathrm{~F}$-FDG could enable the differentiation of stable and rupture-prone plaques by quantifying macrophage density and ${ }^{18}$ F-FDG uptake, an approach that capitalizes on the role of activated macrophages in digesting atheroma fibrous caps and triggering plaque rupture (49). An alternative approach could make use of ultra-small superparamagnetic iron oxide, which tends to selectively accumulate in rupture-prone plaques; in a prospective human study, ultra-small superparamagnetic iron oxide MRI was used to serially monitor the effect of atorvastatin on plaque inflammation (50).

Apoptosis of smooth muscle cells or macrophages within a plaque, causally linked to plaque rupture, is another promising

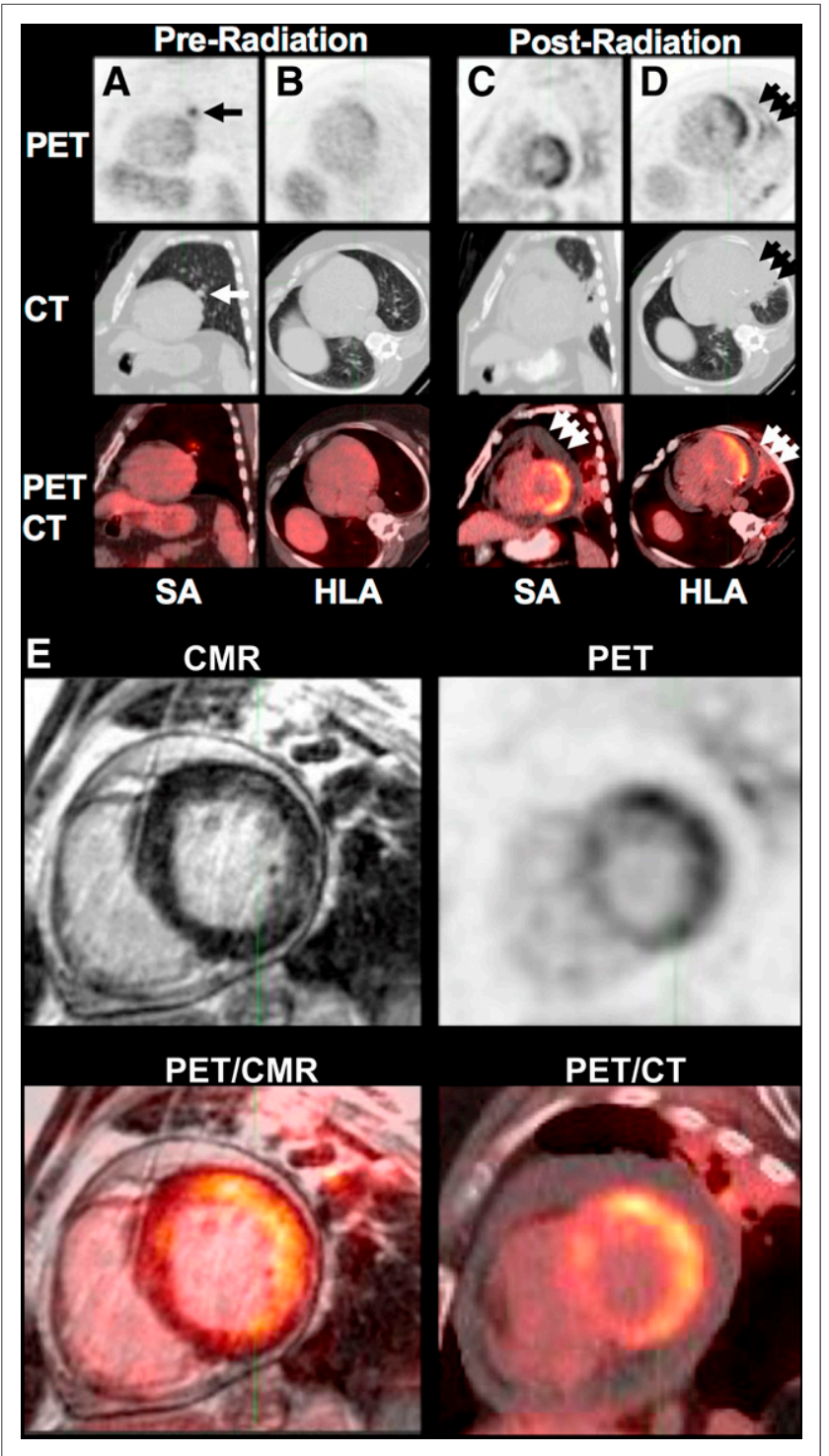

FIGURE 4. Radiation-induced pneumonitis, pericarditis, and myocarditis in patient with cancer of left upper lung near left ventricular lateral wall. (A and B) Before surgical resection and RT, cancer (arrows) is seen on PET and CT. (C and D) About 6 mo after treatment, medium-sized pericardial effusion (C, arrows) and pneumonitis changes ( $D$, arrows) can be seen, along with possible focal ${ }^{18} \mathrm{~F}-\mathrm{FDG}$ uptake along left ventricular lateral wall on PET/CT. (E) Fibrotic changes can be seen on CMR; patchy late gadolinium enhancement and focally increased uptake can be seen along anterolateral and inferolateral wall segments on CMR and PET/ $\mathrm{CT}$, respectively; and pericardial effusion can also be seen on CMR and PET/CT. SA = short axis; HLA = horizontal long axis. 
imaging target. Specifically, the translocation of phosphatidylserine to the external surface of the cell membrane is a targetable, early event occurring in apoptotic cells. For this reason, ${ }^{99 \mathrm{~m}} \mathrm{Tc}-$ labeled annexin V, a plasma protein with high affinity for phosphatidylserine, has been tested in a pilot study to detect cellular apoptosis associated with unstable human carotid plaques (51). Applying this technology to doxorubicin-treated patients, imaging with ${ }^{99 m}$ Tc-HYNIC-annexin $\mathrm{V}$ demonstrated the ability to detect dose-dependent cell death before echocardiography (52). Future prospective studies on larger cohorts will help determine its true sensitivity and specificity.

Although these imaging procedures could potentially revolutionize cardiovascular risk stratification algorithms for cardiooncology patients, they have not yet been studied specifically in RT populations; translation of their findings therefore remains to be determined. Nevertheless, as our understanding of the key molecular determinants of RT-induced CVD grows, the use of molecular imaging specifically tailored to the biomolecular targets identified by -omics approaches could ultimately create a platform for personalized, multidisciplinary cardio-oncologic care.

\section{CONCLUSION}

Cardiotoxic cancer therapies continue to contribute to the global burden of CVD. Further resources should be invested in the pursuit of a more personalized approach to clinical management, centered on deep phenotyping with -omics. Molecular imaging represents a unique component of this potential strategy for diagnostics and surveillance, enabling precise exploitation of underlying pathophysiology. Trends toward individualized medicine will be a critical feature of future paradigms of evidence-based, riskspecific precision medicine in cardio-oncology, ultimately reducing cardiovascular morbidity and mortality in a growing population of cancer patients and survivors.

\section{DISCLOSURE}

Research was supported by the National Center for Advancing Translational Sciences of the National Institutes of Health (TL1TR001880) and R01 HL118018. No other potential conflict of interest relevant to this article was reported.

\section{REFERENCES}

1. Miller KD, Siegel R, Lin C, et al. Cancer treatment and survivorship statistics, 2016. CA Cancer J Clin. 2016;66:271-289.

2. Leopold JA, Loscalzo J. Emerging role of precision medicine in cardiovascular disease. Circ Res. 2018;122:1302-1315.

3. Zamorano JL, Lancellotti P, Rodriguez Muñoz D, et al. 2016 ESC position paper on cancer treatments and cardiovascular toxicity developed under the auspices of the ESC committee for practice guidelines: the task force for cancer treatments and cardiovascular toxicity of the European Society of Cardiology (ESC). Eur Heart J. 2016;37:2768-2801.

4. Cardinale D, Colombo A, Lamantia G, et al. Anthracycline-induced cardiomyopathy: clinical relevance and response to pharmacologic therapy. J Am Coll Cardiol. 2010;55:213-220.

5. Yancy CW, Jessup M, Bozkurt B, et al. ACCF/AHA guideline for the managements of heart failure: executive summary - a report of the American College of Cardiology Foundation/American Heart Association Task Force on practice guidelines. Circulation. 2013;128:1810-1852.

6. Fang N, Jiang M, Fan Y. Ideal cardiovascular health metrics and risk of cardiovascular disease or mortality: a meta-analysis. Int J Cardiol. 2016;214: 279-283.

7. Cardinale D, Sandri M, Colombo A, et al. Prognostic value of troponin I in cardiac risk stratification of cancer patients undergoing high-dose chemotherapy. Circulation. 2004;109:2749-2754.
8. Sandri MT, Salvatici M, Cardinale D, et al. N-terminal pro-B-type natriuretic peptide after high-dose chemotherapy: a marker predictive of cardiac dysfunction? Clin Chem. 2005;51:1405-1410.

9. Ky B, Putt M, Sawaya H, et al. Early increases in multiple biomarkers predict subsequent cardiotoxicity in patients with breast cancer treated with doxorubicin, taxanes, and trastuzumab. J Am Coll Cardiol. 2014;63:809-816.

10. Finkelman BS, Putt M, Wang T, et al. Arginine-nitric oxide metabolites and cardiac dysfunction in patients with breast cancer. J Am Coll Cardiol. 2017; 70:152-162.

11. Blanco JG, Sun C, Landier W, et al. Anthracycline-related cardiomyopathy after childhood cancer: role of polymorphisms in carbonyl reductase genes-a report from the Children's Oncology Group. J Clin Oncol. 2012;30:1415-1421.

12. Visscher H, Ross C, Rassekh S, et al. Validation of variants in SLC28A3 and UGT1A6 as genetic markers predictive of anthracycline-induced cardiotoxicity in children. Pediatr Blood Cancer. 2013;60:1375-1381.

13. Visscher H, Ross C, Rassekh S, et al. Pharmacogenomic prediction of anthracycline-induced cardiotoxicity in children. J Clin Oncol. 2012;30:1422-1428.

14. Armenian SH, Ding Y, Mills G, et al. Genetic susceptibility to anthracyclinerelated congestive heart failure in survivors of haematopoietic cell transplantation. Br J Haematol. 2013;163:205-213.

15. Wells QS, Veatch O, Fessel J, et al. Genome-wide association and pathway analysis of left ventricular function after anthracycline exposure in adults. Pharmacogenet Genomics. 2017;27:247-254.

16. Stanton SE, Ward MM, Christos P, et al. Pro1170 Ala polymorphism in HER2neu is associated with risk of trastuzumab cardiotoxicity. BMC Cancer. 2015; $15: 267$.

17. Oatmen KE, Toro-Salazar O, Hauser K, et al. Identification of a novel microRNA profile in pediatric cancer patients treated with anthracycline chemotherapy. Am J Physiol Heart Circ Physiol. August 24, 2018 [Epub ahead of print].

18. Leger KJ, Leonard D, Nielson D, de Lemos JA, Mammen PP, Winick NJ. Circulating microRNAs: potential markers of cardiotoxicity in children and young adults treated with anthracycline chemotherapy. J Am Heart Assoc. 2017;6: e004653.

19. Ohyama K, Tomonari M, Ichibangase T, et al. A toxicoproteomic study on cardioprotective effects of pre-administration of docetaxel in a mouse model of Adriamycin-induced cardiotoxicity. Biochem Pharmacol. 2010;80:540-547.

20. Stérba M, Popelova O, Lenco J, et al. Proteomic insights into chronic anthracycline cardiotoxicity. J Mol Cell Cardiol. 2011;50:849-862.

21. Beer LA, Kossenkov AV, Liu Q, et al. Baseline immunoglobulin E levels as a marker of doxorubicin- and trastuzumab-associated cardiac dysfunction. Circ Res. 2016;119:1135-1144.

22. Thavendiranathan P, Grant A, Negishi T, Plana J, Popović Z, Marwick T. Reproducibility of echocardiographic techniques for sequential assessment of left ventricular ejection fraction and volumes: application to patients undergoing cancer chemotherapy. J Am Coll Cardiol. 2013;61:77-84.

23. Plana JC, Galderisi M, Barac A, et al. Expert consensus for multimodality imaging evaluation of adult patients during and after cancer therapy: a report from the American Society of Echocardiography and the European Association of Cardiovascular Imaging. Eur Heart J Cardiovasc Imaging. 2014;15:1063-1093.

24. Schwartz RG, McKenzie W, Alexander J, et al. Congestive heart failure and left ventricular dysfunction complicating doxorubicin therapy: seven-year experience using serial radionuclide angiocardiography. Am J Med. 1987;82:11091118.

25. Romond EH, Jeong J, Rastogi P, et al. Seven-year follow-up assessment of cardiac function in NSABP B-31, a randomized trial comparing doxorubicin and cyclophosphamide followed by paclitaxel (ACP) with ACP plus trastuzumab as adjuvant as adjuvant therapy for patients with node-positive, human epidermal growth factor receptor 2-positive breast cancer. J Clin Oncol. 2012;30:3792-3799.

26. Narayan HK, French B, Khan A, et al. Noninvasive measures of ventriculararterial coupling and circumferential strain predict cancer therapeutics-related cardiac dysfunction. JACC Cardiovasc Imaging. 2016;9:1131-1141.

27. Mor-Avi V, Lang R, Badano L, et al. Current and evolving echocardiographic techniques for the quantitative evaluation of cardiac mechanics: ASE/EAE consensus statement on methodology and indications endorsed by the Japanese Society of Echocardiography. Eur J Echocardiogr. 2011;12:167-205.

28. Negishi K, Negishi T, Hare J, Haluska B, Plana J, Marwick T. Independent and incremental value of deformation indices for prediction of trastuzumab-induced cardiotoxicity. J Am Soc Echocardiogr. 2013;26:493-498.

29. Jordan JH, Todd R, Vasu S, Hundley W. Cardiovascular magnetic resonance in the oncology patient. JACC Cardiovasc Imaging. 2018;11:1150-1172.

30. Lightfoot JC, D'Agostino R, Hamilton C, et al. Novel approach to early detection of doxorubicin cardiotoxicity by gadolinium-enhanced cardiovascular magnetic resonance imaging in an experimental model. Circ Cardiovasc Imaging. 2010;3:550-558. 
31. Jordan JH, D'Agostino R, Hamilton C, et al. Longitudinal assessment of concurrent changes in left ventricular ejection fraction and left ventricular myocardial tissue characteristics after administration of cardiotoxic chemotherapies using T1-weighted and T2-weighted cardiovascular magnetic resonance. Circ Cardiovasc Imaging. 2014;7:872-879.

32. Hundley WG, Jordan J. When left ventricular extracellular volume fraction changes after anthracyclines: is it due to a change in the numerator, denominator, or both? JACC Cardiovasc Imaging. 2018;11:1056-1058.

33. Drafts BC, Twomley KM, D'Agostino R Jr, et al. Low to moderate dose anthracycline-based chemotherapy is associated with early noninvasive imaging evidence of subclinical cardiovascular disease. JACC Cardiovasc Imaging. 2013;6:877-885.

34. Hardenbergh PH, Munley MT, Bentel GC, et al. Cardiac perfusion changes in patients treated for breast cancer with radiation therapy and doxorubicin: preliminary results. Int J Radiat Oncol Biol Phys. 2001;49:1023-1028.

35. Lind PA, Pagnanelli R, Marks LB, et al. Myocardial perfusion changes in patients irradiated for left-sided breast cancer and correlation with coronary artery distribution. Int J Radiat Oncol Biol Phys. 2003;55:914-920.

36. Marks LB, Yu X, Prosnitz RG, et al. The incidence and functional consequences of RT-associated cardiac perfusion defects. Int J Radiat Oncol Biol Phys. 2005; 63:214-223

37. Yu X, Prosnitz R, Zhou S, et al. Symptomatic cardiac events following radiation therapy for left-sided breast cancer: possible association with radiation therapyinduced changes in regional perfusion. Clin Breast Cancer. 2003;4:193-197.

38. Bravo PE, Bengel FM. The role of cardiac PET in translating basic science into the clinical arena. J Cardiovasc Transl Res. 2011;4:425-436.

39. Groarke JD, Mamon HJ, Nohria A, Hainer J, Di Carli MF, Killoran J. Negative correlation between coronary flow reserve and mean radiation dose to the heart [abstract]. Int J Radiat Oncol Biol Phys. 2014;90(suppl):S718.

40. Yan R, Song J, Wu Z, et al. Detection of myocardial metabolic abnormalities by ${ }^{18} \mathrm{~F}-\mathrm{FDG} \mathrm{PET} / \mathrm{CT}$ and corresponding pathological changes in beagles with local heart irradiation. Korean J Radiol. 2015;16:919-928.

41. Lee WW, Marinelli B, van der Laan AM, et al. PET/MRI of inflammation in myocardial infarction. J Am Coll Cardiol. 2012;59:153-163.

42. Folco EJ, Sheikine Y, Rocha VZ, et al. Hypoxia but not inflammation augments glucose uptake in human macrophages: implications for imaging atherosclerosis with ${ }^{18}$ fluorine-labeled 2-deoxy-D-glucose positron emission tomography. $J \mathrm{Am}$ Coll Cardiol. 2011;58:603-614.

43. Evans JD, Gomez DR, Chang JY, et al. Cardiac ${ }^{18} \mathrm{~F}$-fluorodeoxyglucose uptake on positron emission tomography after thoracic stereotactic body radiation therapy. Radiother Oncol. 2013;109:82-88.

44. Boutagy $\mathrm{NE}, \mathrm{Wu} \mathrm{J}$, Cai $\mathrm{Z}$, et al. In vivo reactive oxygen species detection with a novel positron emission tomography tracer, ${ }^{18} \mathrm{~F}$-DHMT, allows for early detection of anthracycline-induced cardiotoxicity in rodents. JACC Basic Transl Sci. 2018;3:378-390

45. Guimarães SL, Brandão S, Andrade L, et al. Cardiac sympathetic hyperactivity after chemotherapy: early sign of cardiotoxicity? Arq Bras Cardiol. 2015;105: 228-234.

46. Dos Santos MJ, da Rocha ET, Verberne HJ, da Silv ET, Aragon DC, Junior JS. Assessment of late anthracycline-induced cardiotoxicity by ${ }^{123} \mathrm{I}$-mIBG cardiac scintigraphy in patients treated during childhood and adolescence. J Nucl Cardiol. 2017;24:256-264.

47. Galper SL, Yu JB, Mauch PM, et al. Clinically significant cardiac disease in patients with Hodgkin lymphoma treated with mediastinal irradiation. Blood. 2011;117:412-418.

48. Dolgin E, Using DNA. Radiation therapy gets personal. Science. 2016;353: 1348-1349.

49. Stary HC, Blankenhorn DH, Chandler AB, et al. A definition of the intima of human arteries and of its atherosclerosis-prone regions: a report from the Committee on Vascular Lesions of the Council on Arteriosclerosis, American Heart Association. Arterioscler Thromb. 1992;12:120-134.

50. Tang TY, Howarth SP, Miller SR, et al. The ATHEROMA (Atorvastatin therapy: effects on reduction of macrophage activity) Study. J Am Coll Cardiol. 2009;53: 2039-2050.

51. Kietselaer BL, Reutelingsperger CP, Heidendal GA, et al. Noninvasive detection of plaque instability with use of radiolabeled Annexin A5 in patients with carotid-artery atherosclerosis. $N$ Engl J Med. 2004;350:1472-1473.

52. Gabrielson KL, Mok GS, Nimmagadda S, et al. Detection of dose response in chronic doxorubicin-mediated cell death with cardiac technetium $99 \mathrm{~m}$ Annexin V single-photon emission computed tomography. Mol Imaging. 2008;7: $132-138$. 